\title{
THE INDEPENDENCE RATIO OF REGULAR GRAPHS
}

\author{
BÉLA BOLLOBÁS
}

\begin{abstract}
As a special case of a general result, it is shown that there are cubic graphs of arbitrarily large girth with independence ratio less than 6/13.
\end{abstract}

A set $I$ of vertices of a graph $G=(V, E)$ is said to be independent if no two vertices of $I$ are joined by an edge of $G$. The maximal number of vertices in an independent set is the (vertex) independence number $\beta_{0}(G)$ and $\beta_{0}(G) /|G|$ is the independence ratio of $G$. (Here $|G|$ denotes the order of $G$, that is, the number of vertices of $G$. For the notation and general background, see [3].) The independence ratio is of great interest, not least because of its close relationship to the chromatic number. An immediate corollary of a classical theorem of Brooks [6] is that if a graph has maximal degree $\Delta=\Delta(G)$ and does not contain a $K^{\Delta+1}$, a complete graph of order $\Delta+1$, then its independence ratio is at least $1 / \Delta$. The condition that $G$ does not contain a $K^{\Delta+1}$ is clearly necessary; it is about the weakest condition expressing the fact that $G$ is "not too dense". Lately several authors have given lower bounds for the independence ratio under other "sparseness" conditions (see [1], [2], [8]-[11]). The sparseness of a graph is usually measured by its girth, the minimal length of a cycle. Let $i(\Delta, g)$ be the infimum of the independence ratio of graphs with maximum degree $\Delta$ and girth at least $g$. In this notation Staton [9] proved that $i(\Delta, 4) \geqslant 5 /(5 \Delta-1)$, and so in view of an example of Fajtlowicz [8] we have $i(3,4)=i(3,5)=5 / 14$. Furthermore, concerning cubic graphs of large girth, Hopkins and Staton [10] showed that $\lim _{g \rightarrow \infty} i(3, g)>7 / 18$.

Our aim is to give upper bounds for $i(\Delta, g)$. In particular, we shall show that $i(3, g)$ is bounded away from $\frac{1}{2}$, which seemed the natural value for $\lim _{g \rightarrow \infty} i(3, g)$ and, in fact, $i(3, g)<6 / 13$ for every $g$. In order to do this one has to show that there are cubic graphs of large girth without many independent vertices. We shall not construct such graphs but show their existence by probabilistic methods. Probabilistic methods have been more and more in use since Erdős [7] tackled a similar problem over twenty years ago. However, the proof of our main result has become possible because of a very recent probabilistic model of regular graphs [5]. We state the theorem in its sharpest, though rather unattractive, form; later we deduce from it some more appealing and slightly weaker results.

THEOREM 1. Let $\Delta>3$ be a natural number and suppose that $0<\alpha<1$ satisfies

$$
\begin{aligned}
\alpha\{\Delta \log 2+\log (1 / \alpha)\}+ & (2-\alpha)(\Delta-1) \log (2-\alpha) \\
& +(1-\alpha) \Delta \log (1-\alpha)^{-1}<2(\Delta-1) \log 2 .
\end{aligned}
$$

Received by the editors February 4, 1980 and, in revised form, December 17, 1980.

1980 Mathematics Subject Classification. Primary 05 C99. 
Then for every $g$ there is a $\Delta$-regular graph of girth at least $g$ whose independence ratio is less than $\alpha / 2$. In particular, $i(\Delta, g) \leqslant \alpha / 2$.

Proof. Let $W=\cup_{j=1}^{2 n} W_{j}$ be a fixed set of $2 \Delta n$ labelled vertices, with $\Delta$ vertices in each group $W_{j}$. A configuration $F$ is a partition of $W$ into $\Delta n$ unordered pairs, called the edges of $F$. Clearly there are

$$
N=N(\Delta n)=\left(\begin{array}{c}
2 \Delta n \\
2
\end{array}\right)\left(\begin{array}{c}
2 \Delta n-2 \\
2
\end{array}\right) \cdots\left(\begin{array}{l}
2 \\
2
\end{array}\right) /(\Delta n) !=\frac{(2 \Delta n) !}{(\Delta n) ! 2^{\Delta n}}
$$

configurations. Let $\Omega$ be the set of all $N$ configurations. The set $\Omega$ can also be viewed as a discrete probability space in which every configuration has the same probability $N^{-1}$. Denote by $\Omega^{*}$ the set of all configurations not containing an edge joining vertices of the same class $W_{j}$ or two edges joining the same two classes. The set $\Omega^{*}$ is closely related to the set $\mathcal{G}$ of $\Delta$-regular graphs with vertex set $\left\{W_{1}, W_{2}, \ldots, W_{2 n}\right\}$. Indeed, given $F \in \Omega^{*}$, define a graph $\phi(F) \in \mathcal{G}$ by joining $W_{i}$ to $W_{j}$ if and only if the configuration $F$ contains an edge joining a vertex in $W_{i}$ to a vertex in $W_{j}$. Clearly $\phi\left(\Omega^{*}\right)=\mathcal{G}$. In fact, for each $G \in \mathcal{G}$ we have $\left|\phi^{-1}(G)\right|=$ $(\Delta !)^{2 n}$ so $\left|\Omega^{*}\right|=|\mathcal{G}|(\Delta !)^{2 n}$, though we shall not need this. What we do need is that, as shown in [5],

$$
P\left(\Omega^{*}\right) \rightarrow e^{-\left(\Delta^{2}-1\right) / 4} \text { as } n \rightarrow \infty .
$$

We shall show that for large $n$ there are $\Delta$-regular graphs on $n$ vertices with at most $\log n$ cycles of length at most $g$ and with at most $\alpha n-n^{1 / 2}$ independent vertices. We shall do this by proving that most configurations have analogous properties and using the fact that the configurations in $\Omega^{*}$ have probability bounded away from 0 . We can then produce a regular graph of degree $\Delta$, with no cycles of length at most $g$ such that every set of $\alpha n$ vertices spans at least one edge, by suitably modifying graphs of the type first described. As a matter of fact, we could invoke a somewhat stronger result from [5] concerning the distribution of the number of short cycles in a random regular graph. However, since that way we would end up with the same result, we prefer the present proof which is more self-contained.

An $l$-cycle $(l \geqslant 3)$ of a configuration is a set of $l$ edges, say $\left\{e_{1}, e_{2}, \ldots, e_{l}\right\}$, such that $e_{i}$ joins $W_{j_{i-1}}$ to $W_{j_{i}}$ for some $l$ classes $W_{j_{1}}, W_{j_{2}}, \ldots, W_{j_{1}}=W_{j_{0}}$. Denote by $Z_{l}(F)$ the number of $l$-cycles of a configuration $F$. Given a fixed $l$, what is the expectation of $Z_{l}(F)$ ? Note that $N(\Delta n-l)$ configurations contain the edges of a given $l$-cycle and there are $(2 l)^{-1}(2 n)_{l}(\Delta(\Delta-1))^{l}$ ways of selecting the edges of an $l$-cycle. Here, as in the sequel, $(a)_{b}=a(a-1) \cdots(a-b+1)$. Hence

$$
\begin{aligned}
E\left(Z_{l}\right) & =(2 l)^{-1}(2 n)_{l}(\Delta(\Delta-1))^{l} N(\Delta n-l) / N(\Delta n) \\
& =\frac{(\Delta(\Delta-1))^{l}}{2 l}(2 n)_{l} \frac{(\Delta n)_{l}}{(2 \Delta n)_{2 l}} 2^{l} \rightarrow \frac{(\Delta-1)^{l}}{2 l}
\end{aligned}
$$

as $n \rightarrow \infty$. This implies that for every fixed $g$ we have

$$
P\left(\sum_{l=3}^{g} Z_{l} \geqslant \log n\right) \rightarrow 0
$$

as $n \rightarrow \infty$. 
Now we turn to the sets of independent vertices. Put $s=s(n)=\left\lfloor\alpha n-n^{1 / 2}\right\rfloor=$ $\beta n$. Note that $\beta=\beta(n) \rightarrow \alpha$ as $n \rightarrow \infty$. Denote by $S(F)$ the number of $s$-sets $\left\{W_{j_{1}}\right.$, $W_{j_{2}}, \ldots, W_{j_{s}}$ \} for which $U=\cup_{i=1}^{s} W_{j_{i}}$ spans no edge of $F$.

Let us calculate the expectation of $S$. Denote by $R$ the number of configurations in which $U=\cup_{i=1}^{s} W_{j_{i}}$ spans no edge. There are $s \Delta$ edges incident with the vertices in $U$. Selecting these edges one by one, we see that there are $(2 n \Delta-s \Delta)_{s \Delta}$ choices for these edges. Then these $s \Delta$ edges are incident with $2 s \Delta$ vertices. The remaining $2 n \Delta-2 s \Delta$ vertices can be partitioned into pairs in $N(n \Delta-s \Delta)$ ways. Hence

$$
R=(2 n \Delta-s \Delta)_{s \Delta} N(n \Delta-s \Delta)
$$

Since there are $\left(\begin{array}{c}2 n \\ s\end{array}\right) s$-sets $\left\{W_{j_{1}}, W_{j_{2}}, \ldots, W_{j_{s}}\right\}$,

$$
\begin{aligned}
E(S) & =\left(\begin{array}{c}
2 n \\
s
\end{array}\right) R / N(\Delta n)=\left(\begin{array}{c}
2 n \\
s
\end{array}\right)(2 n \Delta-s \Delta)_{s \Delta} \frac{(2 n \Delta-2 s \Delta) !}{(n \Delta-s \Delta) !} \frac{(n \Delta) !}{(2 n \Delta) !} 2^{s \Delta} \\
& =\frac{(2 n) !}{s !(2 n-s) !} \frac{((2 n-s) \Delta) !}{((n-s) \Delta) !} \frac{(n \Delta) !}{(2 n \Delta) !} 2^{s \Delta} .
\end{aligned}
$$

At this stage it is inevitable to use Stirling's formula: $m !=e^{\gamma}(2 \pi m)^{1 / 2}(m / e)^{m}$, where $(12 m+1)^{-1}<\gamma=\gamma(m)<(12 m)^{-1}$. Note that the product of the contributions of the factors $e^{\gamma}(2 \pi m)^{1 / 2}$ is at most $C n^{-1 / 2}$, where $C$ is independent of $n$. Furthermore, writing $s=\beta n, 2 n-s=(2-\beta) n,(2 n-s) \Delta=(2-\beta) \Delta$, etc., it is easily seen that the exponents of $n, e$ and $\Delta$ are all 0 . By calculating the exponents of $2, \beta, 1-\beta$ and $2-\beta$, we find that

$$
E(S)<C n^{-1 / 2} 2^{-((2-\beta) \Delta-2) n} \beta^{-\beta n}(1-\beta)^{(\beta-1) \Delta n}(2-\beta)^{(2-\beta)(\Delta-1) n} .
$$

Our condition (1) guarantees that if $n$ is sufficiently large then the product of the last four factors is less than 1 so $E(S)<C n^{-1 / 2}$. Consequently,

$$
P(S \geqslant 1) \rightarrow 0 \text { as } n \rightarrow \infty \text {. }
$$

Now we have all the ingredients needed to find a graph with the required properties. Since by (2) the configurations in $\Omega^{*}$ have probability bounded away from 0 , relations (3) and (4) imply that almost all configurations in $\Omega^{*}$ have fewer than $\log n$ cycles of length at most $g$ and contain at most $\beta n=\left\lfloor\alpha n-n^{1 / 2}\right\rfloor$ independent vertices. Let $F_{0} \in \Omega^{*}$ be such a configuration and put $G_{0}=\phi\left(F_{0}\right)$. Then $G_{0}$ is a $\Delta$-regular graph of order $2 n$ containing at most $\log n$ cycles of length at most $g$. Let $G_{1}$ be obtained from $G_{0}$ by omitting one edge from each cycle of length at most $g$. This graph $G_{1}$ has maximum degree $\Delta$. Furthermore, as $G_{0} \operatorname{did}$ not have $\beta n$ independent vertices, $G_{1}$ does not have $\beta n+\lfloor\log n\rfloor<\lfloor\alpha n\rfloor$ independent vertices. Consequently, $G_{1}$ has girth at least $g$, maximum degree $\Delta$ and independence ratio less than $\lfloor\alpha n\rfloor /(2 n) \leqslant \alpha / 2$. Finally by joining together several copies of $G_{1}$, we obtain a $\Delta$-regular graph with the required properties.

For $\Delta=3$ Theorem 1 is easily seen to have the following consequence.

COROLLARY 2. There are cubic graphs of arbitrarily large girth with independence ratio less than $0.459065<157 / 342<6 / 13$. In particular, $i(3, g)<6 / 13$ for every g. 
For a general $\Delta$ relation (1) implies a rather pleasant bound for the independence ratio.

COROLlary 3. $i(\Delta, g)<2(\log \Delta) / \Delta$ for every $g$.

Proof. A crude computer check shows that the assertion is true for all $\Delta<e^{3 e}$, say. (In fact, for $\Delta<8$ the bound is trivial.) Suppose now that $\Delta>e^{3 e}$ and set $\alpha=2(\log \Delta) / \Delta$. Note that

$$
\log (1 / \alpha)<\log \Delta-\log \log \Delta, \quad-(1-\alpha) \log (1-\alpha)<\alpha-\alpha^{2} / 2
$$

and

$$
(2-\alpha) \log (2-\alpha)<2 \log 2-(1+\log 2) \alpha+\alpha^{2} / 4+\alpha^{3} / 8
$$

These inequalities show that (1) holds if

$$
-\alpha(\log \log \Delta-1-\log 2)-\alpha^{2} / 4+\alpha^{3}(\Delta-1) / 8<0 .
$$

As $\Delta>e^{3 e}$, this inequality does hold, so by Theorem $1, i(\Delta, g)<\alpha / 2=$ $2(\log \Delta) / \Delta$, as claimed.

In conclusion let us note a consequence of Corollary 3 concerning the chromatic number, maximum degree and girth. It is proved in [4] that for every $k$ and $g$ there is a graph of chromatic number $k$, maximum degree $16 e k(1+\log k)$ and girth $g$. As a consequence of Corollary 3 , the constant $16 e$ can be improved to 2 .

COROLlaRY 4. For every $g$ there is a graph of maximum degree $\Delta$, girth $g$ and chromatic number at least $\Delta / 2(\log \Delta)$.

Proof. The product of the chromatic number and independence ratio is at least 1.

I am grateful to the referee for his valuable comments.

\section{REFERENCES}

1. M. O. Albertson, B. Bollobás and S. Tucker, The independence ratio and maximum degree of a graph, Proc. Seventh South Eastern Conf. on Combinatorics, Graph Theory and Computing (Louisiana State Univ., Baton Rouge, La., 1976), Utilitas Math., Winnipeg, Man., 1976, pp. 43-50.

2. M. O. Albertson and J. P. Hutchinson, The maximum size of an independent set in a nonplanar graph, Bull. Amer. Math. Soc. 81 (1975), 554-555.

3. B. Bollobás, Extremal graph theory, London Math. Soc. Monographs, No. 11, Academic Press, London and New York, 1978.

4. , Chromatic number, girth and maximum degree, Discrete Math. 24 (1978), 311-314.

5 . $\ldots$ A probabilistic proof of an asymptotic formula for the number of regular graphs, European $\mathrm{J}$. Combinatorics 1 (1980), 311-316.

6. R. L. Brooks, On colouring the nodes of a network, Proc. Cambridge Philos. Soc. 37 (1941), 194-197.

7. P. Erdös, Graph theory and probability, Canad. J. Math. 11 (1959), 34-38.

8. S. Fajtlowicz, The independence ratio for cubic graphs, Proc. Eighth South Eastern Conf. on Combinatorics, Graph Theory and Computing (Louisiana State Univ., Baton Rouge, La., 1977), Utilitas Math., Winnipeg, Man., 1977, pp. 273-277.

9. , On the size of independent sets in graphs, Proc. Ninth South Eastern Conf. on Combinatorics, Graph Theory and Computing, Utilitas Math., Winnipeg, Man., 1978, pp. 269-274.

10. G. W. Hopkins and W. Staton, Girth and independence ratio (to appear).

11. W. Staton, Some Ramsey-type numbers and the independence ratio, Trans. Amer. Math. Soc. 256 (1979), 353-370.

Department of Pure Mathematics and Mathematical Statistics, University of Cambridge, Cambridge, Great Britain 